importance of its abnormal functions in differentiated lymphocytes, there is however no question. Leder was one of several speakers who last week drew attention to the demographic importance of Burkitt's lymphoma in, for example, West Africa.

\section{Longer list}

ROBERT WEINBERG, much involved with the characterization of the activated form of the H-ras oncogene, now has another. Last week, Weinberg described the isolation from a chemically initiated neuroblastoma in rats of an oncogene different from those already known.

Neither the sequence of the new gene nor the function of its product is known as yet. Indeed, the 85,000 molecular weight protein identified on the neuroblastoma cell surfaces may not be coded for directly by the oncogene, but could in principle be a secondary product thereof.

But the protein and the epidermal growth factor receptor are cross reactive, suggesting similarity falling short of identity.

So how many more oncogenes will be found? Weinberg reckons that the next three or four years will bring a further dozen. Obviously the effort will yield diminishing returns, but nobody can know how long the list will be.

Already the list is long enough for classification to be meaningful. Weinberg would divide them into transforming oncogenes (such as the ras genes) and the immortalizing genes (such as myc). And the effects on cells are similarly categorized-some (e.g. sis) allow cells to make their own growth factors, some (erb-B) alter growth factor receptors and other (H-ras) may change the regulation of cell biochemistry.

\section{Binary toxin}

Coupling cytotoxic drugs to antibodies has during the past few years been a commonly favoured strategy for killing specific cells. Dr J. Uhr explained last week his strategy for the development of immunotoxins from the natural product ricin-a constituent of the castor bean. The protein molecule consists of two chains (A and B) which are separated within cells, whereupon A becomes lethal (one molecule, one cell).

Uhr's objective is to couple ricin, and ricin A chains, separately to immunoglobin molecules raised against specific antibodies on circulating B-cells. The objective is the high cell specificity that would promise the avoidance of sideeffects in therapy. A spontaneously arising B-cell clone is being used as a test system for telling whether the effects of A-chains might be potentiated by the manipulation of intracellular conditions or even the use of ricin B-chains also tagged to immunoglobin molecules.

\section{AIDS}

\section{Bad news, good news}

ROBERT Gallo spread a sense of urgency last week about the HTLV group of retroviruses, and especially about HTLV-III, which he and his associates have done most to establish as the agent of AIDS (autoimmune deficiency syndrome). Since the publication of the evidence for the identification of the virus earlier in the summer, complications have arisen.

Gallo's bad news, derived from nucleotide mapping and sequencing, is that HTLV-III may vary considerably in its nucleotide sequence from one patient to another; whether this will affect the outlook for the preparation of vaccines will depend on whether the antigenic sites are affected. The news that AIDS has been recognized among adults with only heterosexual partners, and that (according to some reports) up to 5 per cent of blood offered for transfusion in the United States may be contaminated, suggests that the virus may already be more widespread among the human population than its overt manifestation implies.

The good news is that Gallo and others have cloned the gene, and that its nucleotide sequence is nearly complete. The development of diagnostic tests for AIDS is also well advanced-so much so that physicians are already worrying what they will tell their patients when they have concrete information to give them. And, small comfort though it is, the association of HTLV-III with AIDS, and the prevalence of the disease, is such that Gallo was able to say last week that "we no longer need an animal model for AIDS".

Why should a virus whose effect on the $T$ cells it infects is to kill them feature among oncogenes? Gallo's sober answer is that HTLV-III, like the two other human $\mathrm{T}$-cell viruses now known, is a retrovirus with similar specificity, with HTLV-I now emerging as the agent of the adult T-cell leukaemias (ATL). The more memorable way of putting it is the rhetorical question "Why not use HTLV-III to treat the leukaemias and HTLV-I to cure AIDS?" (The suggestion will not work because different "subsets of subsets of T cells" are responsible for the deficiencies of the immune system that characterize the two groups of diseases.)

Gallo's obsession is to see a vaccine in trials and eventually in general use. Like everybody else, he is infuriated by the knowledge that as things are, it is possible only to treat the adventitious aspects of the syndrome, Kaposi's sarcoma or whatever. The evidence, as he sees it, that the disease is spreading beyond the groups of male homosexuals and haemophiliacs dependent on injected Factor VIII makes the search more urgent.

\title{
Some success with interferon
}

Success in the use of interferon in the treatment of serious cancers is beginning to trickle in. That was the burden of the report by $\mathrm{Dr}$ J. Gutterman of his own group's use of interferon, both alpha and gamma interferon and naturally occurring (and thus heterogeneous) and genetically-engineered material. But the development of therapeutic regimes is painfully slow.

Gutterman described the use of alpha interferon in a group of 27 patients with chronic myeloid leukaemia (CML) in which the underlying defect is a translocation from chromosomes 9 to 22. Only 3 of the 27 patients had falled to go into remission after the administration of interferon, although the question cannot yet be regarded as resolved, given the long duration (3.2 years average) of the chronic phase. But Gutterman says it is encouraging that in many of the patients treated, the proportion of cells with abnormal chromosomes in bone marrow had been markedly reduced, in some cases apparently to zero.

Gutterman also described the successful treatment with gamma interferon of $\mathbf{1 0}$ (out of 11) patients suffering from hairy cell leukaemia, and the generally successful amelioration of Kaposi's sarcoma (an

\section{terferon.}

Even so, the development of effective means of using genetically-engineered and naturally occurring interferon will be an enormous task. Alpha and beta interferon (which share the same cell receptors) do not potentiate each other, but alpha and gamma interferon are mutually synergistic, which argues for appropriate mixtures, not yet defined.

Even more daunting is the need somehow to distinguish between the constitutional genetic variants of three types of interferon specified by the human genes. Clinically, for the time being, this problem has been left on one side.

On the side-efiects of interferon therapy, Gutterman says that the chief efiect seems to be a loss of "executive function", a lassitude, which psychologists describe as a reversible biochemical lobotomy.

With the need to distinguish between all the possible variants of interferon, Gutterman considers there is an urgent need to define various types of cancer in terms of "early molecular events" so as to be able to make use of accumulating knowledge of the role of interferon and of other lymphokines in the physiology of body cells. 